early tests on motor-cycle crash-helmets was shown and specimens illustrated the protection afforded by helmets and the distribution of the injuries of motorcyclists.

The bulk of the meeting was concerned with the results of accidents and diminution of their effects on people; little attention was given to the prevention of motor-car accidents by methods within the control of the vehicle driver. The papers and discussion will be published in the Annals of Occupational Hygiene.

\section{N. Davies}

\title{
MECHANISMS OF VIRUS INFECTIONS
}

\begin{abstract}
A DISCUSSION meeting of the Royal Society, arranged by Prof. Wilson Smith, on the subject of "Mechanisms of Virus Infections", was held on November 2. Owing to the large numbers attending, the meeting was transferred, by courtesy of Sir Lawrence Bragg, to the lecture hall of the Royal Institution. The president of the Royal Society, Sir Howard Florey, took the chair.

In his introduction, Prof. Wilson Smith traced briefly the progress of virological knowledge from the early almost metaphysical questioning into the nature of viruses to the present wealth of information regarding their chemical constitution, structure and intracellular behaviour. $\mathrm{He}$ wondered, however, whether these particular aspects were not in danger of being over-emphasized at the expense of more traditional problems relating to virus disease. The natural history of any virus disease is intimately related to the survival cycle of the virus concerned and, in the metazoon host, this cycle is necessarily complex. The route of infection and primary lodgement, paths and mechanisms of spread through the host, replication and ultimate release must be considered at the various levels of the cell, the intact host and the host population. It was with this in mind that the present programme had been built up around the concept of the survival cycle.
\end{abstract}

Prof. A. W. Downie (University of Liverpool) opened the discussion with a paper on "Mechanisms of Virus Entry and Spread within the Host (Animal Viruses)". Although a rapid survey of the various routes of infection known to occur was given to illustrate the extent of the subject, and problems associated with the question of neural spread in such diseases as poliomyelitis, herpes and pseudo-rabies were briefly discussed, the paper was centred on the pathogenesis of disease in the pox group of viruses. Fenner's detailed work on ectromelia has laid a pattern which serves as a standard of reference for investigations with other members of this group, and a study of the highly lethal rabbit pox infection shows that it follows the same general course. The main difference is that the stages of primary and secondary lodgement with final involvement of the skin, and of primary and secondary viræmia, are less well defined in rabbit pox than in mouse pox. Despite thorough investigation of animals infected by the intra-nasal route, no primary lesion is identifiable although virus can be readily demonstrated in the tissues of the nasal passages and trachea. The blood contains virus as early as the second day after infection and thereafter remains positive. Clinical disease develops on the fourth day. The finding that provoked the greatest interest and discussion was that animals do not become infectious by contact until the fifth or sixth day, at least $24 \mathrm{hr}$. after the onset of clinical disease, a finding closely similar to that in human smallpox. Following intranasal infection, virus may be isolated from the tissues of the upper respiratory tract in rabbit pox, and smallpox virus is presumed to invade by this route in the majority of human cases. Why then is the patient not infective during the incubation period? Dr. C. H. Cadman (Scottish Horticultural Research Institute) dealt with the same subject in its relation to plant viruses and pointed out that there is an essential difference in the problems involved, since plant viruses are unable to effect entry into the tissues without external aid. Trauma is an essential prerequisite to infection and is usually inflicted in Nature by insect vectors. In the laboratory the necessary damage is achieved by rubbing the leaf, but the inefficiency of this procedure may be gauged from the fact that about $10^{6}$ infective particles are necessary in order to produce a lesion. Further, since the plant does not possess conveniently isolated internal organs which can be segregated by dissection and to which the virus can track, spread of virus cannot be followed as in the animal host. It is probable that virus spreads in the translocation stream, but if so the form of the virus at this time is not known. Since certain viruses are apparently capable of dispensing permanently with their protein eoat, it is possible that spread within the plant occurs in the form of ribonucleic acid. The possible role of plasmodesmata and the problem presented by the fact that virus appears to spread through callus tissue cuitures just as fast as through intact plant tissues were also discussed.

It was clear from both these papers, in their different contexts, that there is a long way to go before the problems of virus invasion of host tissues can be considered to be satisfactorily explained.

"Intracellular Replication Mechanisms" were discussed by Prof. M. Stoker (University of Glasgow), who considered the subject from the point of view of the interrelationship of virus growth to cell growth. The types of interrelationship exemplified by bacteriophage infection are: (1) the extreme virulence found in the $T$-even phage where adsorption of the protein coat is sufficient to cause immediate death of the cell with breakdown of deoxyribonucleic acid; (2) intermediate virulence where the host cell is not immediately killed but cell division is prevented; and (3) the avirulent prophage relationship. In the case of animal viruses, infection usually results in death of the cell, and the difficulties in demonstrating noncytopathic infection of cells were discussed in detail. For proof it is necessary to study isolates of single cells and demonstrate, first, release of virus and, second, cell division in that order.

Prof. Stoker concluded that there is as yet no satisfactory evidence for infections of this type with normally cytopathogenic viruses, but this has recently been acquired by Rubens with the Rous sarcoma virus so that this virus, which is not normally cytopathogenic, is the only one for which the formal criteria have been satisfied. In the case of polyoma virus, either cytopathic effect or cell transformation may follow infection in vitro; but in the latter case, it has not proved possible to demonstrate virus in the 
transformed cells and these remain super-infectable with polyoma virus, albeit with difficulty. It must be concluded that persistence of the virus is not necessary for these cells to retain their character once transformation has occurred.

Thus three types of non-cytopathogenic relationship appear to be possible. In the case of the Rous virus both virus and cell multiply with the intermittent release of infective virus; in lysogenic bacteria both multiply together but virus is not released without induction and death of the cell occurring, and finally with polyoma the virus may induce a cell transformation without itself persisting.

Dr. A. Isaaes (National Institute for Medical Research) considered "Interference Phenomena" as a further aspect of intracellular behaviour. Many interference phenomena have now been shown to be mediated by interferon, and the properties of this substance were described. It is a protein of molecular weight 63,000 which is produced by all vertebrate tissues so far tested. All viruses examined have been found to provoke production of interferon and all are to some extent susceptible to its action. In its protective action it possesses some specificity for the species in which it is produced. It is non-toxic at viral inhibitory concentrations and does not inhibit cell division. In infected cells it suppresses the production of viral nucleic acid, possibly through its inhibitory activity against oxidative processes providing the energy for synthesis. As might be expected, therefore, it is found that viruses depending on oxidative processes for their replication are more strongly inhibited than those which do not have this dependence.

The possible role of interferon in recovery from virus infections was discussed and some interesting speculations added on the possibility that the action of interferon in virus infections is incidental to its role in the normal cell. Dr. Isaacs suggested that its function might in fact be that of excluding extraneous ribonucleic acid of whatever nature. It is not formed during the first third of chick embryonic life when ribonucleic acid might be acting as a vital intermediary in the processes of morphogenesis, but thereafter is formed in increasing amounts in response to the appropriate stimulus. It might be that its formation in later embryonic life is a mechanism for the protection of the tissues from unwanted morphogenetic stimuli. This suggestion aroused some argument and it was pointed out that interferon does not exclude viral ribonucleic acid from the cell but apparently suppresses its formation.

The last paper on cellular relationships of virus was given by Prof. G. Belyavin (University College Hospital Medical School, London) on "Latency in Virus Infections". Prof. Belyavin treated the subject from a purely theoretical point of view and demonstrated by means of a mathematical model that in any system where persistence of infection depends on a balance between the rate of replication of virus and the rate of replication of cells, an impossibly critical state would exist. Such a system would inovitably load either to the virus gaining the upper hand with eventual destruction of the whole cell population, or to cell multiplication outstripping virus multiplication with the elimination of the latter. The assumptions on which the model was constructed did not include variation in the susceptibility of the cell population or the role taken by such substances as interferon. The intervention of some such factors would be essential if a stable balance between virus and host cells were to be achieved.
Epidemiological factors in relation to animal and plant virus diseases respectively were presented by Prof. F. J. Fenner (Australian National University) and Dr. L. Broadbent (Glasshouse Crops Research Institute). These two papers provided an interesting comparison of the problems in these two fields, the more so as Prof. Fenner confined himself to a discussion of myxomatosis, a vector-transmitted disease. A comparison of the Brazilian, Californian and Australian viruses in rabbits native to those regions suggests that, in the establishment of the disease in Nature, natural selection acts not on the virus as such but on the factor of transmissibility. Thus the three types of virus are capable of infecting the Californian rabbit $S$. buchmani, but whereas the attenuated Australian strain fails to give detectable virus in the skin lesions and the South American strain gives a titre of only $10^{4} / \mathrm{gm}$., the Californian virus achieves a titre of $10^{8} / \mathrm{gm}$. and only the last is found to be transmissible by mosquitoes. In Australia since 1952, the original virus, which killed close on 100 per cent of infected animals, has been largely replaced by less virulent variants. The fall in mortality has not, however, been extreme or progressive, and the dominant strains have for some years remained in the 'grade III' category in which mortality is about 90 per cent ; but since the infected rabbits live longer, the virus is available for transmission for a period of about 15 days as against 5 days with the highly virulent strains. However, the increase in the numbers of genetically resistant rabbits may ultimately favour the more virulent virus.

The highly complex interplay of factors governing the epidemiology of myxomatosis was reflected in the field of plant viruses. Dr. Broadbent dealt with the epidemiology of plant virus diseases in wider terms, but the main interest in his paper lay not in the artificial spread of viruses by human agency or even by contact, but in the intricacies governing its transmission by vectors. While the nature and static habits of plants eliminate the complications arising from behaviour patterns, they equally introduce factors specific to plant epidemiology. Thus, while it is easy to understand that the size of plants and their position relative to each other and to sources of infection external to the crop must influence spread of infection, the results of the microclimate within the crop are more subtle. If plants are closely spaced, the transfer of vectors will be facilitated; but this may be offset by the development of temperature and humidity conditions within the crop which are not tolerable to them. The vector will then be confined to the new shoots which may contain little virus. Similarly, plants are in general more susceptible to infection when young, so that climatic influences on the time of greatest prevalence of the vector may determine whether or not infection is widespread irrespective of whether the vector is particularly numerous. Despite these factors, which have no real counterpart in the animal virus field, there are more similarities than differences apparent in the epidemiology of plant and animal viruses and Dr. Broadbent's paper served to illustrate some of these. For example, some viruses, such as potato virus $Y$, can be picked up in a few seconds feeding and immediately spread to uninfected hosts. These virusos do not persist in the vector for more than a few hours. Others, such as potato leaf roll, require prolonged foeding to infect the vector, cannot be transmitted before the lapse of a latent period, and thereafter persist in the vector 
for many weeks. This contrast is very similar to that between myxomatosis and yellow fever.

Finally, the damage which virus infection inflicts on the host was discussed by Dr. J. C. N. Westwood (Miorobiological Research Establishment, Porton) in relation to the intact host and by Dr. Alison Newton (University of Cambridge) at the cellular level. Dr. Westwood discussed "Virus Toxicity" and its possible role in the production of disease and ultimately death in fatal virus infections. The term 'virus toxicity' was originally coined to cover the production, by massive doses of virus, of lesions or death in susceptible or non-susceptible animals without the occurrence of multiplication of the virus. The toxic factor has always been inseparably associated with the virus particle itself. Since virus disease is normally initiated by small doses of virus and accompanied by multiplication, it has seemed unlikely that 'toxicity' could be involved; and the latter has always been regarded as a freak laboratory phenomenon unassociated with production of disease. It was suggested that this concept might be wrong. Experiments were described which showed that the toxicity of rabbit pox virus is associated with a partial cycle of replication, as is the toxicity of other viruses in which such partial cycles have been sought. Death in rabbit pox is preceded by the abrupt onset of shock, with some evidence of increased capillary permeability, and the clinical picture can best be explained by the presence of some toxic factor in the blood. During the four days preceding death in acutely fatal infections, the virus concentration in the blood may rise to as high as $10^{6}$ infective units $/ \mathrm{ml}$. and similar concentrations oceur in fatal smallpox and herpes infections in man. The cytotoxic effect of such virus concentrations on the capillary endothelium could be the cause of the increase in capillary permeability and so precipitate the animal into shock. Since no toxic factor other than the virus has ever been demonstrated to result from cell-virus interaction, the possible role of direct virus toxicity in disease must be seriously considered.

Damage to the intact host depends on the damage produced at the cellular level, and Dr. Newton considered the "Biochemical Aspects of Virus Infectivity" in relation to modification of cellular metabolism. The main modifications are found first in the production of new virus and second in changes in the nucleic acid and protein synthesis. Viruses which multiply in the cytoplasm make less use of the cell metabolism-for example, vaccinia virus does not affect the metabolism of deoxyribonucleic acid-and such viruses probably produce their damaging effects by competition for metabolites. Those which multiply in the nucleus produce a more profound effect, since they must not only compete for metabolites but must also overcome the normal control processes of the cell. Thus pre-labelling of the cellular deoxyribonucleic acid with tracer thymidine shows that herpes virus causes a rapid breakdown of cell deoxyribonucleic acid probably due to increased deoxyribonuclease activity similar to the increased ribonuclease activity stimulated by poliovirus. It also stimulates synthesis of deoxyribonucleic acid. These changes must damage the control mechanisms of the cell, and interfere with synthetic activities in the host. In some viruses, such as encephalomyocarditis, viral ribonucleic acid is elaborated in the nucleus and protein in the cytoplasm. It is probable that in these instances cytoplasmic protein metabolism is governed by viral ribonucleic acid which migrates from the nucleus. The production of cell damage does not depend on completion of the multiplication cycle of the virus; for example, with poliovirus, herpes and rabbit pox viruses, inhibition of infective virus production does not prevent cell destruction. It was suggested that cell damage results from the release of lysozomos, disruption of the controlling mechanism of the cell, competition for metabolites and disturbance of metabolic balance.

The meeting was successful in provoking lively discussion and considerable disagreement on nearly every point raised by the main speakers. Dr. Sanders, in summing up, congratulated the participants on avoiding all reference to infective nucleic acids, and he also stressed the gulf which still exists between academic virology and virology as applied to disease. J. C. N. Westwood

\section{COTTON-GROWING IN NIGERIA}

$A^{a}$ GRICULTURAL progress relies on the pure Tciences, on engineering and on economics. problems have been resolved into their scientific components. Resolution is one of the functions of husbandry investigation. Recognition of the nature and importance of this function has come only within the past three decades. In this same period the other function of husbandry investigation has also been developed, namely, improvement of accepted practices in raising erops and livestock. Cultivations, drainage, sowing, grazing, housing of stock and other day-to-day matters were not looked on, formerly, as problems amenable to study : they were matters for the farmer's experience and tradition. Modern husbandry investigation seeks to improve all working practices by studying their underlying purposes and interpreting their effects.

That physical environment is primarily important for agriculture is now an axiom, though some costly flouting of it is yet fresh in memory. It is largely through husbandry investigation that scientific attention has been brought to its present intensity in such matters as rainfall characteristics, solar radiation, air temperature, shade, wind and moistureholding capacity of soils.

Some highly successful husbandry investigations on cotton have recently been reported* from the Ministry of Agriculture Research Station at Samaru, Northern Nigeria.

Past work at the Station and in its area of interest had revealed a discouraging situation. Local yields were $150-200 \mathrm{lb}$. of seed cotton an acre. Late sowing partly accounted for this, but earlier sowing in experimental plots gave only $240 \mathrm{lb}$. Assisted by fertilizers and by early planting the maximum yield was $600 \mathrm{lb}$. At these yields insecticidal protection did not pay. Yield in a bad year was only half of that in a good one and in any small field good and bad patches markedly influenced growth and yield. An improved variety of cotton, bred at the Station,

* Lawes, D. A., Emp. J. Exp. Agric., 29, 116 (1961). 